\title{
Влияние электрон-фононного взаимодействия и облучения $\gamma$-квантами на обратные токи кремниевых фотодиодов
}

\author{
(C) С.В. Булярский ${ }^{1}$, А.В. Лакалин ${ }^{1}$, М.A. Сауров ${ }^{2}$ \\ ${ }^{1}$ Институт нанотехнологий микроэлектроники Российской академии наук, \\ 119991 Москва, Россия \\ ${ }^{2}$ Национальный исследовательский университет „Московский институт электронной техники“, \\ 124498 Москва, Зеленоград, Россия \\ E-mail: bulyar2954@mail.ru
}

Поступила в Редакцию 3 июня 2020 г.

В окончательной редакции 3 августа 2020 г.

Принята к публикации 25 августа 2020 г.

Исследованы вольт-амперные характеристики кремниевых фотодиодов до и после облучения $\gamma$-квантами энергией $1.25 \mathrm{MэВ} \mathrm{и} \mathrm{дозой} \mathrm{облучения} 0.5$ МРад. Установлено, что обратные токи определяются механизмом Пула-Френкеля в сильном электрическом поле с влиянием электрон-фононного взаимодействия. Разработана методика и рассчитаны параметры электрон-фононного взаимодействия, описываемого конфигурационной однокоординатной моделью, из обратных вольт-амперных характеристик. Сделано предположение, что в результате облучения $\gamma$-квантами образуются центры дивакансии кремния с кислородом, которые и определяют обратные токи фотодиодов.

Ключевые слова: обратная вольт-амперная характеристика, эффект Пула-Френкеля, электрон-фононное взаимодействие, облучение $\gamma$-квантами, центры дивакансии кремния с кислородом.

DOI: 10.21883/FTP.2021.01.50389.9455

\section{1. Введение}

Взаимодействие радиационного излучения с полупроводниками сопровождается возникновением дефектов кристаллической решетки: вакансий и межузельных атомов основного вещества, а также более сложных дефектов вторичного дефектообразования [1,2]. Такие дефекты существенно изменяют основные физические свойства полупроводников, в первую очередь время жизни неосновных носителей заряда и обратные токи приборов [3-6]. К настоящему времени накоплена достаточно обширная информационная база об образовании радиационных дефектов в кремнии и кремниевых структурах, а также проводились исследования параметров глубоких центров различными методами [1-8]. Анализ этих работ показывает, что дефекты, которые образуются в результате радиационного облучения, в первую очередь оказывают влияние на величину обратных токов $p-n$-переходов [9].

$\mathrm{C}$ точки зрения диффузионной теории протекания тока в $p-n$-переходе обратный ток не зависит от приложенного напряжения [10]. Его рост при облучении прибора связан с изменением времени жизни при облучении [1]. Энергия активации температурной зависимости диффузионного тока должна быть равной энергии ширины запрещенной зоны полупроводника, однако экспериментальные работы показывают, что это не так, и энергия меньше ширины запрещенной зоны $[9,11,12]$. Это связано с тем, что в большинстве случаев обратный ток является суммой диффузионного тока и тока генерации [13], причем вклад последнего преобладает [9]. Механизм протекания тока можно определить, изучив его зависимость от температуры.
Если экспериментальное значение энергии активации тока меньше энергии ширины запрещенной зоны, но больше ее половины, то обратный ток определяется процессом генерации электронов и дырок через уровни глубоких центров и не должен зависеть от напряжения смещения на $p-n$-переходе [14]. Генерация электронов и дырок происходит в области пространственного заряда (ОПЗ) $p$-n-перехода, где существует достаточно сильное электрическое поле, которое оказывает влияние на величину тока. В большинстве работ, которые исследуют обратные вольт-амперные характеристики полупроводниковых приборов, это явление остается без внимания, а в других влияние приложенного напряжения объясняется эффектом Пула-Френкеля $[15,16]$. Коэффициент Пула-Френкеля имеет четко определенное теоретическое значение. Это значение должно быть рассчитано и сопоставлено с его экспериментальной величиной для того, чтобы доказать существование данного эффекта. Однако коэффициент Пула-Френкеля, вычисленный из экспериментальных результатов, может быть больше теоретического, что связано с влиянием электрон-фононного взаимодействия $[9,17]$. Это влияние в некоторых случаях может быть достаточно сильным, например, обратный ток кремниевых тиристоров вначале монотонно возрастает с увеличением приложенного напряжения, а затем становится экспоненциальным [18]. Без учета электрон-фононного взаимодействия эту зависимость объяснить нельзя.

В данной работе изучаются вольт-амперные характеристики кремниевых $p-n$-переходов до и после облучения $\gamma$-квантами изотопа ${ }^{60}$ Со. На этом примере приводится методика обработки обратных вольт-амперных характеристик, определяются параметры электрон- 
фононного взаимодействия и строится конфигурационно-координатная диаграмма, показывающая изменение энергии активации рекомбинационного центра в сильном электрическом поле.

\section{2. Экспериментальные результаты}

Экспериментально были исследованы кремниевые фотодиоды, изготовленные на основе низколегированного кремния $n$-типа с удельным сопротивлением $\sim 500$ Ом ·см, кристаллы которого были выращены методом бестигельной зонной плавки. Для создания тонкой $p$-области пластина кремния окислялась при температуре $950^{\circ} \mathrm{C}$ в атмосфере сухого кислорода до толщины пленки 950А̊. Через полученную пленку тонкого диэлектрика производилась ионная имплантация бора с дозой 8 мкКл/см². Отжиг введенной примеси в атмосфере азота происходил в два этапа, при температурах 900 и $700^{\circ} \mathrm{C}$ для активации примеси и минимизации дислокационных дефектов в структуре. Активная область диода имела площадь $5.4 \cdot 10^{-2} \mathrm{~cm}^{2}$ и была окружена охранным кольцом.

Проводилось измерение электрических характеристик диодов, затем они подвергались облучению и эти измерения повторялась заново. Облучение образцов осуществлялось $\gamma$-квантами, которые испускались источником на основе ${ }^{60} \mathrm{Co}$ (энергия $\gamma$-квантов $E \approx 1.25 \mathrm{MэB).} \mathrm{Доза} \mathrm{облучения} \mathrm{составила} 0.5$ МРад. Измеренные вольт-емкостные характеристики были линейны в координатах $C^{-2}=f(U)$. Это указывает на то, что $p$-n-переход является резким и примеси вблизи перехода распределены равномерно. Концентрация примесей, вычисленная по результатам измерения, составляла $(5.9 \pm 0.2) \cdot 10^{11} \mathrm{~cm}^{-3}$ до облучения и $(5.5 \pm 0.2) \cdot 10^{11} \mathrm{~cm}^{-3}$ после, что связано с компенсацией материала при возникновении радиационных дефектов.

Были проведены измерения вольт-амперных характеристик кремниевых фотодиодов в интервале температур $-20-+60^{\circ} \mathrm{C}$ как до облучения, так и после облучения. Для измерений BAX на постоянных токах использовался анализатор полупроводниковых приборов В1500А фирмы Keysight. Прибор оптимизирован для измерений слаботочных сигналов вплоть до токов $10^{-15}$ А и оснащен специальными экранированными триаксиальными выводами с функцией компенсации сверхмалых токов. Входное сопротивление прибора (> 100 ТОм) обеспечивает при измерениях самый минимальный уровень вносимых искажений и ошибок в тестируемые схемы в этом классе приборов.

Обратные вольт-амперные характеристики до и после облучения при различных температурах приведены на рис. 1 в зависимости от корня из напряженности электрического поля. Приведена средняя напряженность электрического поля, вычисленная в результате измерения изменения емкости при приложении обратного

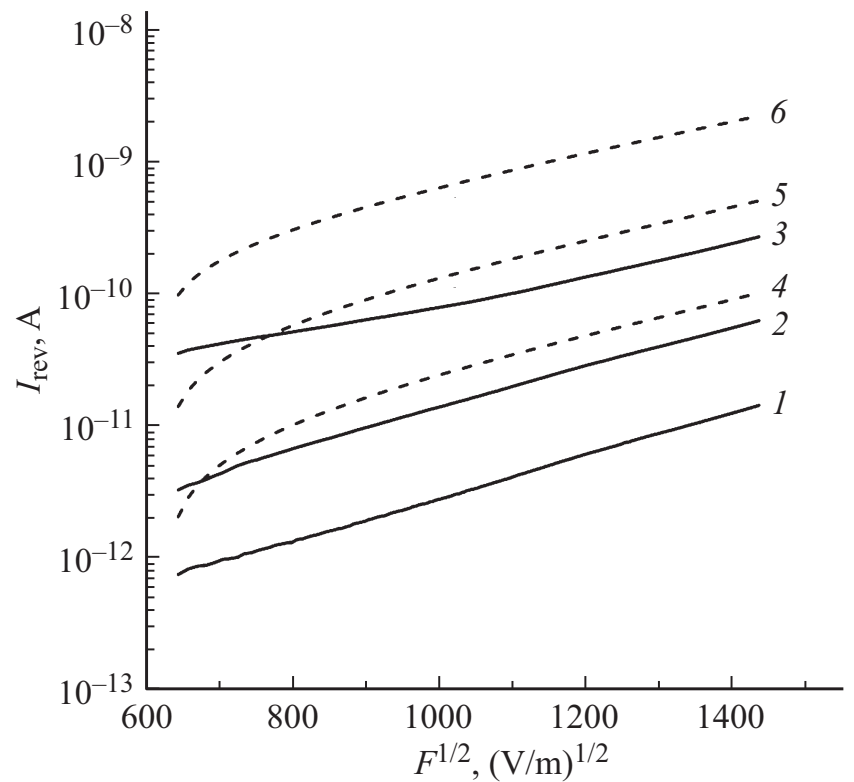

Рис. 1. Обратные вольт-амперные характеристики, построенные в координатах Пула-Френкеля до облучения $(1-3)$ и после облучения $(4-6)$, измеренные при температурах ${ }^{\circ} \mathrm{C}$ : $1,4-0 ; 2,5-20 ; 3,6-40$.

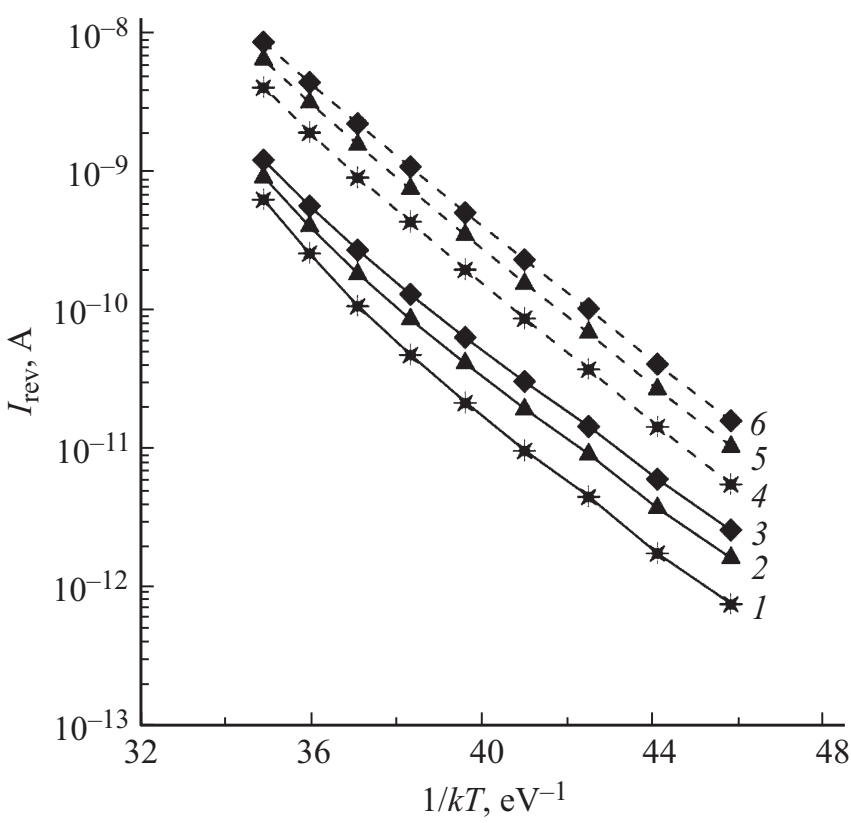

Рис. 2. Зависимость тока фотодиода от обратной температуры для различных напряжений. До облучения (сплошные линии), $\mathrm{B}: 1-10,2-20,3-30$; после облучения (штриховые линии), В: $4-10,5-20,6-30$.

напряжения смещения. Ток возрастал с ростом напряжения, с увеличением температуры и после облучения. Вольт-амперные характеристики, построенные в координатах $\ln (I)=f\left(F^{1 / 2}\right)$ (где $F-$ напряженность электрического поля в ОПЗ диода), были линейны. Эти факты указывают на то, что ток через диод при обратном 


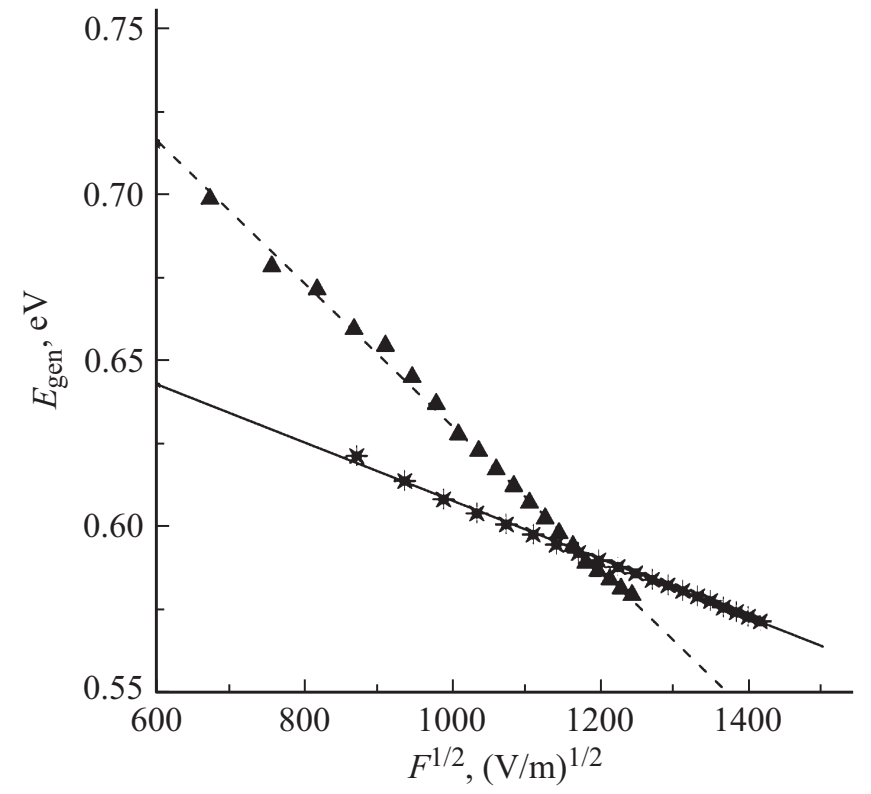

Рис. 3. Изменение энергии активации процесса генерации в зависимости от напряженности электрического поля до облучения (треугольники) и после (звездочки).

смещении обусловлен генерацией и имеет место эффект Пула-Френкеля, который понижает высоту барьера генерации пропорционально корню из напряженности электрического поля. Температурные зависимости обратного тока, построенные в координатах Аррениуса при различных напряжениях обратного смещения на диоде (рис. 2), показывают, что энергия активации процесса генерации зависит от электрического поля (рис. 3), что также является проявлением эффекта Пула-Френкеля.

\section{3. Обсуждение экспериментальных результатов}

При обратном напряжении ОП3 $p-n$-перехода обеднена свободными носителями заряда, равновесие между рекомбинацией и генерацией сдвинуто в сторону генерации. Обратный ток определяется выражением

$$
I_{\mathrm{rev}}=q S \int_{0}^{w} R d x
$$

где $q$ - элементарный заряд, $R$ - скорость генерации, $S$ - площадь $p-n$-перехода. В случае генерации с участием двухзарядного рекомбинационного центра (РЦ) одного типа ееё скорость можно найти из системы кинетических уравнений:

$$
\begin{aligned}
& R_{n}=\frac{d n}{d t}=-c_{n} n\left(N_{t}-n_{t}\right)+e_{n}^{t} n_{t}, \\
& R_{p}=\frac{d p}{d t}=-c_{p} p n_{t}+e_{p}^{t}\left(N_{t}-n_{t}\right),
\end{aligned}
$$

где $N_{t}$ - концентрация глубоких центров, $n_{t}-$ концентрация электронов на них; $e_{n}^{t}$ и $e_{p}^{t}-$ скорости эмиссии электронов и дырок с рекомбинационных центров; $c_{n}$ и $c_{p}$ - коэффициенты захвата электронов и дырок на РЦ.

При вычислении скорости генерации принимается во внимание, что в положении равновесия $R=R_{n}=R_{p}$. С использованием этого равенства из системы (2) получается:

$$
I_{\mathrm{rev}}=q S \int_{0}^{w} \frac{e_{n}^{t}(x) e_{p}^{t}(x) N_{t}(x)}{e_{n}^{t}(x)+e_{p}^{t}(x)} d x
$$

где $\quad e_{n}^{t}=\gamma_{n} c_{n} N_{c} \exp \left(-\left(E_{C}-E_{t}\right) / k_{\mathrm{B}} T\right), \quad e_{p}^{t}=\gamma_{p} c_{p} N_{v}$ $\times \exp \left(-\left(E_{C}-E_{V}\right) / k_{\mathrm{B}} T\right), \gamma_{n}, \gamma_{p}-$ факторы вырождения уровня глубокого центра для электронов и дырок. Данные факторы изменяются в пределах от 0.5 до 2.

Следует отметить, что скорость термической эмиссии зависит от температуры экспоненциально, поэтому если уровень РЦ отличается от середины запрещенной зоны на $(3-5) k_{\mathrm{B}} T$, то, как правило, скорость эмиссии либо электронов, либо дырок намного больше, чем скорость другого перехода. Делается следующее приближение: уровень рекомбинации расположен ближе к зоне проводимости; РЦ равномерно распределены по ОПЗ; скорости термической эмиссии не зависят от электрического поля. Получается простое выражение для обратного тока диода:

$$
I_{\mathrm{rev}}=q S W N_{t} e_{p}^{t}
$$

где $W$ - ширина ОПЗ.

В формулу (4) входит скорость эмиссии дырок, а не электронов, так как величина тока при обратном смещении определяется самым медленным процессом, а именно скоростью эмиссии с большей энергией активации, которая в случае термической генерации через РЦ получается больше половины ширины запрещенной зоны. Поэтому энергия рекомбинационного центра при нулевой напряженности электрического поля $\left(E_{t}(0)\right)$ связана с энергией активации процесса генерации обратного тока $\left(E_{\text {gen }}\right)$ по формуле

$$
E_{t}(0)=E_{g}(0)=E_{\text {gen }} .
$$

Формула (4) показывает, что величина тока генерации при обратном смещении прямо пропорциональна концентрации рекомбинационных центров. Измеренный ток генерации после облучения вырос в 10 раз. Можно предположить, что в 10 раз увеличилась концентрация рекомбинационных центров. Формула (4) в первом приближении правильно описывает температурную зависимость тока (рис. 2). Однако из этой формулы вытекает достаточно слабая корневая зависимость тока от напряжения обратного смещения, которая обусловлена изменением ширины ОПЗ, и из нее не следует, что энергия активации эмиссии дырок зависит от напряжения. Экспериментально измеренный ток (рис. 1), построенный в координатах Пула-Френкеля, имеет экспоненциальную зависимость, что обусловлено изменением вероятности эмиссии от напряженности электрического поля в ОПЗ $p-n$-перехода. 
Параметры центров генерации, которые обусловливают обратный ток до и после облучения

\begin{tabular}{l|c|c|c|c}
\hline $\begin{array}{c}\text { Экспериментальные } \\
\text { и расчетные параметры }\end{array}$ & $\beta_{F e x}$, эB $\cdot \mathrm{cm}^{1 / 2} / \mathrm{B}^{1 / 2}$ & $E_{g}(0)-E_{\text {gen }}(0)$, эB & $S \hbar \omega$, эB & $E_{0}$, эB \\
\hline До облучения & $21.5 \cdot 10^{-4}$ & 0.32 & 0.004 & 0.063 \\
После облучения & $8.7 \cdot 10^{-4}$ & 0.47 & 0.032
\end{tabular}

Понижение высоты потенциального барьера в силу эффекта Пула-Френкеля [9] имеет вид

$$
\Delta E_{t}=\frac{q^{3 / 2}}{\sqrt{\pi \varepsilon \varepsilon_{0}}} \sqrt{F}=\beta_{F T} \sqrt{F} .
$$

Множитель перед корнем из электрического поля, который носит название постоянная Френкеля $\left(\beta_{F T}=q^{3 / 2} / \sqrt{\pi \varepsilon \varepsilon_{0}}\right)$, не зависит от технологических параметров прибора, а определяется только константами. Его величина равна в практических единицах 0.00023 эВ $\cdot \mathrm{cm}^{1 / 2} / \mathrm{B}^{1 / 2}$.

С помощью формулы (6) вычисляется выражение для вольт-амперной характеристики $p-n$-перехода при обратном смещении:

$$
\begin{aligned}
I_{\mathrm{rev}} & =q S e_{p 0}^{t} \int_{0}^{W} N_{t}(x) \exp \left(\frac{\Delta E_{t}(x)}{k T}\right) d x \\
& =q S e_{p 0}^{t} \int_{0}^{W} N_{t}(x) \exp \left(\frac{\beta_{F T} \sqrt{F(x)}}{k T}\right) d x,
\end{aligned}
$$

где $e_{p 0}^{t}-$ скорость эмиссии дырок без учета влияния электрического поля.

Формула (7) учитывает влияние и температуры, и напряженности электрического поля. Энергия активации процесса генерации с учетом эффекта Пула-Френкеля равна:

$$
E_{\text {gen }}(F)=E_{\text {gen }}(0)-\beta_{F T} \sqrt{F},
$$

где $E_{\text {gen }}(0)$ - энергия активации процесса генерации в отсутствие электрического поля. Эта зависимость в координатах $E_{\mathrm{gen}}(F)=f\left(F^{1 / 2}\right)$ имеет вид прямой линии. Такой же вид имеет аппроксимация экспериментальных результатов на рис. 3, что позволяет определить энергию процесса генерации в отсутствие электрического поля и экспериментальное значение константы ПулаФренкеля. Результаты обработки экспериментальных обратных вольт-амперных характеристик приведены в таблице. В нашем случае экспериментальные значения $\beta_{F e x}$ больше, чем теоретическое $\beta_{F T}$ в несколько раз, что связано с электрон-фононным взаимодействием. В работе Тимашева [17] показано, что

$$
\beta_{F e x}=\beta_{F T}\left(1+\frac{E_{0}-S \hbar \omega}{2 S \hbar \omega}\right),
$$

где $S \hbar \omega-$ величина, равная половине тепловыделения, которое сопровождает электрон-фононное взаимодействие; $E_{0}$ - энергия чисто электронного перехода.
Выражение (9) справедливо, когда генерация носителей заряда происходит в результате многофононного процесса и в нем принимает участие только один тип фононов, имеющих некоторую эффективную энергию. Такая модель носит название однокоординатной модели и для нее можно использовать конфигурационнокоординатную диаграмму [19], которая показана на рис. 4. В соответствии с приближением однокоординатной модели для электрон-фононного взаимодействия величина энергии термической генерации равна (рис. 4) [20]:

$$
E_{t}=E_{g}(0)-E_{\mathrm{gen}}(0)=\frac{\left(E_{0}+S \hbar \omega\right)^{2}}{4 S \hbar \omega} .
$$

Значение экспериментальной константы Френкеля $\beta_{F e x}$ и энергии термической активации процесса генерации в отсутствие электрического поля $E_{\mathrm{gen}}(0)$ вычисляется по экспериментальным данным, которые приведены на рис. 3. Затем из совместного решения уравнений (9) и (10) рассчитываются параметры $S \hbar \omega$ и $E_{0}$. Результаты вычислений представлены в таблице. Как видно из таблицы, все параметры после облучения принимают иные значения.

Электрон-фононное взаимодействие, как правило, проявляется сильнее в молекулярных объектах, поэтому можно предположить, что природа центров рекомбинации, которые найдены в данной работе, связаны с молекулярными комплексами вакансии кремния с примесью. Известно, что при больших дозах облучения в

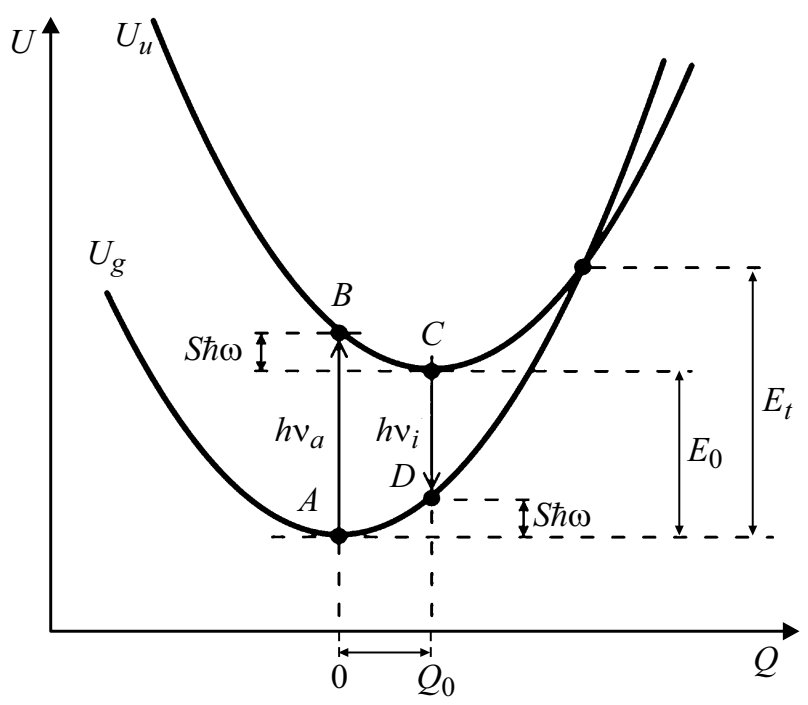

Рис. 4. Конфигурационно-координатная диаграмма. 
кремниевых диодах имеют место процессы, связанные с метастабильностью таких центров и эффектом ЯнаТеллера, для них присущих [19,21], что также является проявлением электрон-фононного взаимодействия.

Прежде чем перейти к анализу возможной природы выявленных центров рекомбинации, следует сделать два замечания. Во-первых, в генерации тока принимают участие локальные состояния вблизи половины ширины запрещенной зоны. Это связано с тем, что скорость генерации спадает экспоненциально с ростом энергии активации процесса. Во-вторых, молекулярные центры кремния являются многозарядными и проявление того или иного зарядового состояния зависит от условий, при которых проходит процесс измерения. При измерении параметров центров рекомбинации методом нестационарной спектроскопии глубоких уровней (DLTS) при каждом акте измерения рекомбинационные центры заполняются путем приложения напряжения к диоду в прямом направлении. При этом измеряются параметры электронного состояния, которые успевают изменять свой заряд за время, равное ширине окна скоростей в эксперименте DLTS. Поэтому сравнение с этими экспериментами надо проводить с пиками, которые расположены в высокотемпературной области DLTS. Также необходимо заметить, что по этим экспериментам трудно судить - является ли центр двух- или многозарядным.

Анализ публикаций по дефектам в кремнии показывает, что энергия активации 0.47 эВ может соответствовать как дивакансии $\left(V_{\mathrm{Si}}\right)_{2}$ [22-26], так и более сложному комплексу $\left(V_{\mathrm{Si}}\right)_{2} \mathrm{O}[22,26-30]$. Рекомбинационный центр с энергией активации 0.32 эВ может быть связан с некоторым неидентифицированным центром $A 5$ [30]. Поэтому можно сделать вывод, что при облучении кремния $\gamma$-квантами концентрация дефектов вакансионного типа увеличивается.

\section{4. Заключение}

В работе развита методика определения параметров центров рекомбинации на основании изучения обратных вольт-амперных характеристик. Данная методика показывает, что имеет место влияние электрон-фононного взаимодействия на процесс генерации обратного тока диодов. Экспериментальные результаты данной работы показывают, что в изучаемых диодах имеются вакансионно-примесные комплексы, включающие кислород. При облучении происходит преобразование одного из комплексов в другой, предположительно, содержащий большее число вакансий кремния, которые образуются при облучении $\gamma$-квантами.

\section{Финансирование работы}

Работа выполнена при поддержке Министерства образования и науки РФ, проект № 0004-2019-0001.

\section{Конфликт интересов}

Авторы заявляют, что у них нет конфликта интересов.

\section{Список литературы}

[1] В.С. Вавилов, Н.А. Ухин. Радиационные эфбеекты в полупроводниках и полупроводниковых приборах (М., Атомиздат, 1969).

[2] H.N. Yeritsyan, A.A. Sahakyan, N.E. Grigoryan, V.V. Harutyunyan, V.M. Tsakanov, B.A. Grigoryan, A.S. Yeremyan, G.A. Amatuni. J. Electron. Mater., 46, 841 (2017). https://doi.org/10.1007/s11664-016-4975-6

[3] B. Biro, G. David, A. Fenyvesi, J.S. Haggerty, J. Kierstead, E.J. Mannel, T. Majoros, J. Molnar, F. Nagy, S. Stoll, B. Ujvari, C.L. Woody. IEEE Trans. Nucl. Sci., 66 (7), 1833 (2019). https://doi.org/10.1109/TNS.2019.2921102

[4] I. Pintilie, E. Fretwurst, G. Lindstrom, J. Stahl. Appl. Phys. Lett., 81 (1), 165 (2002). https://doi.org/10.1063/1.1490397

[5] L.I. Murin, J.L. Lindström, G. Davies, V.P. Markevich. Nucl. Instr. Meth. Phys. Res., Sect. B, 253, 210 (2006). https://doi.org/10.1016/j.nimb.2006.10.029

[6] M. Mikelsen, J.H. Bleka, J.S. Christensen, E.V. Monakhov, B.G. Svensson, J. Härkönen, B.S. Avset. Phys. Rev. B: Condens. Matter, 75, 155202 (2007). https://doi.org/10.1103/PhysRevB.75.155202

[7] E. Fretwurst, G. Lindstrom, J. Stahl, I. Pintilie. Proc. 9th Eur. Symp. Semicond. Detect.: New Develop. Rad. Detect. (Schloss Elmau, Germany, 2002). [Nucl. Instr. Meth. Phys. Res. Sect. A, $512(1-2), 111$ (2003). https://doi.org/10.1016/S01689002(03)01884-9]

[8] R. Pagano, S. Lombardo, F. Palumbo, D. Sanfilippo, G. Valvo, G. Fallica, S. Libertino. Nucl. Instr. Meth. Phys. Res., Sect. A, 767, 347 (2014). https://doi.org/10.1016/j.nima.2014.08.028

[9] S.V. Bulyarskiy. Solid-State Electron., 160, 107624 (2019). https://doi.org/10.1016/j.sse.2019.107624

[10] W. Shockley. Bell System Techn. J., 28, 435 (1949). https://doi.org/10.1002/j.1538-7305.1949.tb03645.x

[11] T. Kimoto, O. Takemura, H. Matsunami, T. Nakata, M. Inoue. J. Electron. Mater., 27 (4), 358 (1998). https://doi.org/10.1007/s11664-998-0415-6

[12] K. Al Abdullah, F. Al Alloush, A. Jaafar, C. Salame. Energy Proc., 36, 104 (2013). https://doi.org/10.1016/j.egypro.2013.07.013

[13] C.T. Sah, R.N. Noyce, W. Shockley. Proc. IRE, 45 (9), 1228 (1957). https://doi.org/10.1109/JRPROC.1957.278528

[14] N.S. Grushko, A.V. Lakalin, A.I. Somov. Semiconductors, 42 (13), 1532 (2008). https://doi.org/10.1134/S1063782608130174

[15] Q. Shan, D.S. Meyaard, Q. Dai, J. Cho, E. Fred Schubert, J. Kon Son, C. Sone. Appl. Phys. Lett., 99, 253506 (2011). https://doi.org/10.1063/1.3668104

[16] M. Musolino, D. van Treeck, A. Tahraoui, L. Scarparo, C. De Santi, M. Meneghini, E. Zanoni, L. Geelhaar, H. Riechert. J. Appl. Phys., 119 (4), 044502 (2016). https://doi.org/10.1063/1.4940949

[17] С.Ф. Тимашев. ФТП, 8, 804 (1974).

[18] V.V.N. Obreja. Solid-State Electron., 44(1), 49 (2000). https://doi.org/10.1016/S0038-1101(99)00208-7 
[19] Л.И. Мурин, В.П. Маркевич, И.Ф. Медведева, L. Dobaczewski. ФТП, 40 (11), 1316 (2006).

http://journals.ioffe.ru/articles/viewPDF/6186

L.I. Murin, V.P. Markevich, I.F. Medvedeva, L. Dobaczewski. Semiconductors, 40 (11), 1282 (2006). https://doi.org/10.1134/S1063782606110066

[20] Физика полупроводниковых преобразователей, под ред. акад. РАН, проф. А.Н. Саурова, чл.-кор. АН Татарстана, проф. С.В. Булярского (М., РАН, 2018).

[21] С.Б. Ластовский, В.П. Маркевич, А.С. Якушевич, Л.И. Мурин, В.П. Крылов. ФТП, 50 (6), 767 (2016). http://journals.ioffe.ru/articles/viewPDF/43202

S.B. Lastovskii, V.P. Markevich, H.S. Yakushevich, L.I. Murin, V.P. Krylov. Semiconductors, 50 (6), 751 (2016). https://doi.org/10.1134/S1063782616060130

[22] P. Pichler. Intrinsic Point Defects, Impurities, and Their Diffusion in Silicon (Springer, 2004). https://doi.org/10.1007/978-3-7091-0597-9

[23] S.D. Brotherton, P. Bradley. J. Appl. Phys., 53, 5720 (1982). https://doi.org/10.1063/1.331460

[24] J.M. Meese, J.W. Farmer, C.D. Lamp. Phys. Rev. Lett., 51, 1286 (1983). https://doi.org/10.1103/PhysRevLett.51.1286

[25] A.M. Frens, M.T. Bennebroek, A. Zakrzewski, J. Schmidt, W.M. Chen, E. Janzen, J.L. Lindstrom, B. Monemar. Phys. Rev. Lett., 72, 2939 (1994). https://doi.org/10.1103/PhysRevLett.72.2939

[26] J.H. Evans-Freeman, A.R. Peaker, I.D. Hawkins, P.Y.Y. Kan, J. Terry, L. Rubaldo, M. Ahmed, S. Watts, L. Dobaczewski. Mater. Sci. Semicond. Process., 3, 237 (2000).

[27] G. Alfieri, E.V. Monakhov, B.S. Avset, B.G. Svensson. Phys. Rev. B: Condens. Matter, 68, 233202 (2003). https://doi.org/10.1103/PhysRevB.68.233202

[28] Z. Pastuovic, I. Capan, R. Siegele, R. Jacimovic, J. Forneris, D.D. Cohen, E. Vittone. Nucl. Instr. Meth. Phys. Res., Sect. B, 332, 298 (2014). https://doi.org/10.1016/j.nimb.2014.02.082

[29] G.F. Chen, Y.X. Li, L.L. Liu, P.J. Niu, S.L. Niu, D.F. Chen. Trans. Nonferrous Met. Soc. Chi., 16, 113 (2006). https://doi.org/10.1016/S1003-6326(06)60155-6

[30] P. Hazdra, V. Komarnitskyy. Mater. Sci. Eng. B, 159-160, 346 (2009). https://doi.org/10.1016/j.mseb.2008.10.008

\section{The effect of electron-phonon interaction and $\gamma$-ray irradiation on the reverse currents of silicon photodiodes}

\author{
S.V. Bulyarskiy ${ }^{1}$, A.V. Lakalin ${ }^{1}$, M.A. Saurov ${ }^{2}$ \\ ${ }^{1}$ Institute of Nanotechnology of Microelectronics, \\ Russian Academy of Sciences, \\ 119991 Moscow, Russia \\ ${ }^{2}$ National Research University of Electronic \\ Technology, \\ 124498 Moscow, Zelenograd, Russia
}

\begin{abstract}
The current-voltage characteristics of silicon photodiodes were studied before and after irradiation with $\gamma$-rays with an energy of $1.25 \mathrm{MeV}$ and an irradiation dose of $0.5 \mathrm{MRad}$. It has been established that the reverse current-voltage characteristic is determined by the Poole-Frenkel mechanism in a strong electric field with the influence of electron-phonon interaction. A technique has been developed and the parameters of the electronphonon interaction of a configuration single-coordinate model have been calculated from reverse current-voltage characteristics. It has been suggested that as a result of irradiation with $\gamma$-rays, divacancy centers of silicon with oxygen are formed, which determine the reverse currents of the photodiodes.
\end{abstract}

Редактор А.Н. Смирнов 